\title{
Natural Deep Eutectic Solvent as Extraction Media for the Main Phenolic Compounds from Olive Oil Processing Wastes
}

\author{
Sonia Bonacci $^{1}$ (D), Maria Luisa Di Gioia ${ }^{2, *}$ (D), Paola Costanzo ${ }^{1, *}$ (D) Loredana Maiuolo $^{3}$ (D), \\ Sofia Tallarico ${ }^{1}$ and Monica Nardi ${ }^{1}$ (D) \\ 1 Dipartimento di Scienze della Salute, (Università Magna Græcia, Viale Europa, 88100-Germaneto CZ), Italy; \\ s.bonacci@unicz.it (S.B.); s.tallarico@unicz.it (S.T.); monica.nardi@unicz.it (M.N.) \\ 2 Dipartimento di Farmacia e Scienze della Salute e della Nutrizione, Edificio Polifunzionale, \\ Università della Calabria, 87030 Arcavacata di Rende, Cosenza \\ 3 Dipartimento di Chimica e Tecnologie Chimiche, Università della Calabria, Cubo 12C, \\ 87036-Arcavacata di Rende (CS), Italy; loredana.maiuolo@unical.it \\ * Correspondence: ml.digioia@unical.it (M.L.D.G.); pcostanzo@unicz.it (P.C.); \\ Tel.: +39-0984-493095 (M.L.D.G.); +39-0961-3694094 (P.C.)
}

Received: 21 May 2020; Accepted: 9 June 2020; Published: 11 June 2020

\begin{abstract}
In this new century, sustainable development challenges chemical sciences to develop new and clean technological processes. The agri-food industry produces significant quantities of waste, raising significant economic and environmental concerns. Food waste valorization using environmentally friendly procedures is of increasing importance. This study describes the use of several Natural Deep Eutectic Solvents (NADESs) for the microwave-assisted extraction (MAE) of valuable bioactive phenolic compounds from olive oil processing wastes. The extracted samples were characterized by liquid chromatography electrospray ionization quadrupole time-of-flight mass spectrometry (LC-ESI-QTOF/MS) analysis and the quantification of the phenolic compounds was performed by HPLC analysis. The obtained data were compared with those obtained using water as the solvent in the same extraction conditions. The extraction process is nontoxic, simple and selective and meets most of the criteria to be considered as a sustainable process, with the solvents arising directly from nature.
\end{abstract}

Keywords: natural deep eutectic solvents; microwave assisted extraction; phenolic compounds; olive oil processing wastes

\section{Introduction}

Every year, the olive oil processing industry produces a great amount of waste, such as twigs, leaves and olive mill wastewater (OMW). The low cost of these kinds of residues favors their application in various processes, varying from use in animal feed or in nutraceutical, pharmaceutical and cosmetic manufacturing. Thus, olive leaves are gaining increasing interest for their use in high-added-value compounds.

It is well established that the leaves of Olea europaea L. contain a quantity of phenolic compounds higher than that present in the fruit or in virgin olive oil: $1450 \mathrm{mg}$ of total phenols/100 $\mathrm{g}$ of fresh leaves [1] against $110 \mathrm{mg} / 100 \mathrm{~g}$ of fruit [2] and $23 \mathrm{mg} / 100 \mathrm{~mL}$ of extra virgin olive oil [3].

In particular, olive leaves have phenolic compounds in common with other plants, but represent a rich source of secondary metabolites, among which secoiridoids and flavonoids stand out. Olive leaves are rich in secoiridoids, especially oleosides, which are specific secoiridoids of the olive trees commonly esterified at the phenolic portion. Oleuropein (Ole), the most abundant bioactive phenol in olive leaf 
extract, and ligstroside are the secoiridoid glucosides that confer organoleptic properties to olive leaves and fruits. The scientific literature available on olive polyphenols is focused mainly on Oleuropein, thanks to its important pharmacological activities [4-8].

The hydrolysis of oleuropein and ligstroside results in the formation a series of degradation products, all of them less hydrophilic than the original secoiridoids [9-11], and therefore more soluble in the oily matrix. In recent years, the biological properties of aglycone forms have been studied [12], in particular oleocanthal ( $p$-HPEA-EDA), the elenolic acid ester of the tyrosol responsible of pharyngeal pungency sensation that occurs when consuming extra virgin olive oil (EVOO) [13], and its hydroxytyrosol derivative analog oleacein (3,4-DHPEA-EDA) [14,15].

After Beauchamp et al. first described the anti-inflammatory properties of oleocanthal as being similar to ibuprofen [14], numerous pharmacological/biological activities were reported for this olive oil secoiridoid derivative and its analog oleacein [15-21]. Unfortunately, only small amounts of either secoiridoid can be obtained from natural matrices (fruits and leaves of the olive tree, OMW, EVOO, etc.), which has prompted researchers to develop different pathways for total and semi-synthesis of oleacin and oleocanthal [22-26].

Hence, the many chemical, agronomical and pharmaceutical positive characteristics of oleuropein, and the possibility of synthesizing the most valuable secoiridoid derivatives starting from this compound alone, together contributed to increasing the interest in the recovery of phenolic compounds from olive leaves (waste product of olive oil industries) with the aim of producing functional foods and nutraceuticals.

Conventionally, the extraction of phenolic compounds from plant leaves and fruits is carried out by maceration, using organic solvents such as ethanol, methanol, dichloromethane, acetone, hexane and ethyl acetate [27]. The yields using these solvents are high, and the product obtained is of good quality. However, these processes require long treatment times and high temperatures, and the solvents used are harmful to human health and the environment; additionally, the extracts, prior to use, must be subjected to solvent removal and purification treatments.

Currently, the development of innovative and environmentally friendly extraction procedures is in increasing demand, as this will be extremely valuable in terms of reducing both the extraction time and the solvent consumption through the application of profitable strategies that are in line with the principles of Green Chemistry [28-30].

In recent years, the concepts of the green economy and eco-sustainability have led to increased attention being paid to deep eutectic solvents (DESs), new solvents that are emerging as a promising and greener alternative to conventional organic solvents [31-35]. DESs represent a new generation of solvents that are mostly based on mixtures of cheap and easily available components: non-toxic quaternary ammonium salts (e.g., choline chloride) and an unbound, naturally derived hydrogen donor (e.g., amides, sugars, alcohols, polyols) [36].

Moreover, the mechanism by which some living beings (beetles, frogs, earthworm, tardigrades, stoneflies, etc.) are able to survive the enormous temperature deviations of some of the most extreme habitats on our planet has recently been discovered. They are able to produce metabolites that, through a process known as vitrification, prevent the crystallization of water, which would lead to cell destruction. These compounds, mainly sugars, organic acids, choline derivatives, or urea, when combined in a certain composition, form a new deep eutectic liquid phase referred to as Natural Deep Eutectic Solvents (NADES) [37].

NADES fully respect the principles of Green Chemistry and are considered "future solvents", because they allow nutraceuticals and food-grade extracts to be obtained $[38,39]$. One of the most important advantages of NADES is the possibility of easily preparing these solvents, together with the large number of combinations that could be applied [40].

Considering the numerous structural possibilities of NADES and the possibility of designing their physical-chemical properties in order to adapt them to different purposes, they can without any doubt be considered "design solvents". Currently, the use of DES as extraction solvents for phenolic 
compounds and more generally for the extraction of natural products for pharmaceutical applications is by far the most studied application [41].

A large number of NADES based on natural compounds (Figure 1), in particular primary metabolites such as sugars, organic acids, urea and quaternary ammonium salts such as choline chloride, have been reported and tested [31,42].<smiles>C[N+](C)(C)CCO</smiles>

choline chloride<smiles>CC(O)C(=O)O</smiles>

lactic acid<smiles>O=C(O)C(O)C(O)C(=O)O</smiles>
tartaric acid<smiles>O=C(O)CC(O)(CC(=O)O)C(=O)O</smiles>

citric acid<smiles>NC(=O)O</smiles><smiles>O=C1O[C@H]([C@H](O)CO)C(O)=C1O</smiles>

ascorbic acid<smiles>O=C[C@H](O)[C@@H](O)[C@@H](O)[C@H](O)CO</smiles>

glucose<smiles>OC[C@H]1O[C@@H](O[C@H]2O[C@H](CO)[C@H](O)[C@]2(O)CO)[C@H](O)[C@@H]1O</smiles>

sucrose<smiles>O=C[C@H](O)[C@H](O)[C@H](O)CO</smiles>

xylose<smiles>O=C(CO)C(O)[C@H](O)[C@@H](O)CO</smiles>

fructose

Figure 1. Chemical structure of some compounds that can be constituents of Natural Deep Eutectic Solvents.

Some authors have studied DES-assisted extraction of phenolic compounds, demonstrating that many compounds are dissolved better than in other traditional and alternative solvents [43-46]. In particular, DES have the ability to donaate and accept protons and electrons, which gives them the ability to form hydrogen bonds, thus increasing their dissolution capacity. Therefore, since microwaveassisted extraction (MAE) has emerged in recent years on account of its numerous advantages (shorter extraction times, reduced solvent consumption, and more effective extraction), we planned to develop a green methodology that envisaged the combined action of some NADESs and the activation of microwaves for the extraction of phenolic compounds from olive leaves and ripe drupes coming from the production of the olive oil.

\section{Materials and Methods}

\subsection{Samples, Standards, and Reagents}

The olive leaves (Coratina cultivar, Olea europaea L.) and the ripened olive drupes (Leccino cultivar) used in this study were provided by CREA-Research center (Rende, CS, (Italy). Samples were randomly collected from several trees and immediately transferred to the laboratory, washed with distilled water, 
and dried under controlled temperature, for $48 \mathrm{~h}$ at $50^{\circ} \mathrm{C}$, until a constant weight was achieved. The ripened olive drupes were ground and stored at $-20^{\circ} \mathrm{C}$ until extraction.

\subsection{NADEs Preparation}

NADESs were fundamentally prepared by heating choline chloride with different hydrogen donors. The components were placed in a round-bottom flask and heated to $80^{\circ} \mathrm{C}$ in a water bath with agitation until a homogeneous liquid was formed. Additionally, an addition of $20 \% v / v$ water in the NADES solutions was performed.

\subsection{Extraction of Phenolic Compounds from Olive Leaves or Ripened Olive Drupes with NADES}

$2 \mathrm{~g}$ of sample was mixed with $5 \mathrm{~mL}$ of NADES in a closed-vessel and the mixture was subjected to microwave (MAE) at $100 \mathrm{~W}$ (time 10 or $30 \mathrm{~min}$ at $80^{\circ} \mathrm{C}$ ). The obtained extract was centrifuged at $1000 \mathrm{rpm}$ for $10 \mathrm{~min}$ and the supernatant was collected. The sample was then filtered under vacuum, diluted with ethanol for the successive analysis by HPLC and LC-ESI-QTOF/MS.

\subsection{Extraction of Phenolic Compounds from Olive Leaves or Ripened Olive Drupes with Water (Control Sample)}

In parallel, with the aim of comparing the extraction efficiency exhibited by the NADES, the same procedure was carried out using water as the solvent.

\subsection{HPLC Analysis}

HPLC analysis was performed using Thermo Scientific (Rodano, MI, Italy) Dionex Ultimate 3000, equipped with a $25 \mathrm{~cm} \times 4.6 \mathrm{~mm}$ Thermo Scientific Hypersil GOLD C18 column packed with $5 \mu \mathrm{m}$ particles. For HPLC separation of the phenolic compounds in DESs, a gradient elution with a mixture of solvents $\mathrm{A}\left(\mathrm{H}_{2} \mathrm{O} /\right.$ trifluoroacetic acid, $\left.\mathrm{pH}=2.46\right)$ and $\mathrm{B}$ (acetonitrile) was used. The column was equilibrated in $95 \%$ solvent $\mathrm{A}$ and $5 \%$ solvent $\mathrm{B}$. The elution flow rate was $1 \mathrm{~mL} \mathrm{~min}^{-1}$ by linearly increasing of solvent B concentration from 5 to $60 \%$ over $17 \mathrm{~min}$, maintained isocratic for $2 \mathrm{~min}$, subsequently increased to $95 \%$ over $6 \mathrm{~min}$, then returned to $5 \%$ over $3 \mathrm{~min}$ and equilibrated for $5 \mathrm{~min}$. The chromatograms were acquired at $280 \mathrm{~nm}$. The instrumentation performance, chromatograms, and initial data processing were carried out with Chromeleon software.

A calibration curve was built using standard solutions of pure oleuropein (2000 ppm), its aglycone form (3,4-DHPEA-EA) (2000 ppm), hydroxytyrosol (2000 ppm), oleacein ((3,4-DHPEA-EDA) (2000 ppm) and demethyloleuropein (2000 ppm) in EtOH; these solutions were then mixed to obtain six standard solutions of $10 \mathrm{ppm}, 25 \mathrm{ppm}, 50 \mathrm{ppm}, 75 \mathrm{ppm}, 100 \mathrm{ppm}$ and $125 \mathrm{ppm}$ in both of phenolic compounds. HPLC analysis gave rise to five regression curves (see Supplementary Material). Each DES extract was diluted in $50 \mu \mathrm{L}$ (in the case of olive leaves) or $500 \mu \mathrm{L}$ (in the case from ripe drupes) of ethanol, which is a miscible solvent in all the deep eutectic solvents used in this study. Ethanol represents the solvent of choice to avoid the formation of acetals from oleacein; $20 \mu \mathrm{L}$ of the diluted extracts were analyzed by HPLC and peaks in the chromatograms were identified by comparison with standards.

\subsection{LC-ESI-QTOF/MS Analysis}

Analyses were carried out using an Agilent 6540 UHD Accurate-Mass Q-TOF LC/MS (Agilent, Santa Clara, CA, USA) fitted with an electrospray ionization source (Dual AJS ESI) operating in positive ion mode. Chromatographic separation was achieved using a $\mathrm{C}_{18} \mathrm{RP}$ analytical column (Poroshell 120 , SB-C18, $50 \times 2.1 \mathrm{~mm}, 2.7 \mu \mathrm{m}$ ) at $40{ }^{\circ} \mathrm{C}$ with an elution gradient from 20 to $35 \%$ of B over $30 \mathrm{~min}$, followed by $35-90 \%$ of $\mathrm{B}$ over $5 \mathrm{~min}$, with $\mathrm{A}$ being $\mathrm{H}_{2} \mathrm{O}(0.1 \% \mathrm{FA})$ and $\mathrm{B} \mathrm{CH}_{3} \mathrm{CN}$. Flow rate was $0.4 \mathrm{~mL} / \mathrm{min}$. The ESI source was operated both in positive ion mode with the following conditions: the fragmentor voltage was set at $120 \mathrm{~V}$, nebulizer gas was set at $35 \mathrm{psig}$, capillary voltage was set at $3500 \mathrm{~V}$, and drying gas flow rate and temperature were set at $10 \mathrm{dm}^{3} / \mathrm{min}$ and $300{ }^{\circ} \mathrm{C}$, respectively. For MS/MS measurements collision energy ramp ranging from 15 to $40 \mathrm{eV}$ to promote fragmentation was used. 
The data were acquired in centroid and profile mode using High Resolution mode (4 GHz). The mass range was set at 50-1000 m/z in MS and MS/MS mode. The data were processed with the MassHunter Workstation QualitativeAnalysis.B.03.01 Software. The Q-TOF-MS was calibrated on a daily basis.

\subsection{Statistical Analysis}

The results are expressed by mean \pm S.E.M. from at least three independent experiments. For statistical comparisons, quantitative data was analyzed by one-way analysis of variance (ANOVA) followed by Tukey-test according to the statistical program SigmaStat1 (Jandel Scientific, Chicago, IL, USA). A $p$-value less than 0.05 was regarded as significant.

\section{Results and Discussion}

\subsection{Screening of the NADESs}

The structure of NADESs determines their chemical and physical properties, and consequently influences the extraction efficiency of biologically active compounds.

Generally, NADESs are formed of natural, biodegradable and economical compounds. For example, choline chloride $(\mathrm{ChCl})$ is cheap (about $150 € / \mathrm{kg}$ ), biodegradable (93\% in 14 days), non-toxic (LD50 $=3500 \mathrm{mg} / \mathrm{kg}$ ) and in addition can be either extracted from biomass or easily obtained from fossil sources. As far as hydrogen bond donors are concerned, the most common ones are urea, carboxylic acids and polyols obtained from renewable sources.

To represent the different "classes" of hydrogen bonds donors typically used in NADES, six different NADESs (Table 1) were chosen based on choline chloride in combination with: urea (NADES-1), glycerol (NADES-2), lactic acid (NADES-3), ethylene glycol (NADES-4), and citric acid (NADES-5).

Table 1. Compositions and abbreviations for the prepared NADESs.

\begin{tabular}{ccccc}
\hline Composition HBA: HBD & Molar Ratio & Water Addition (\%) & Acronym & Physical Aspect Color \\
\hline ChCl: Urea & $1: 2$ & 0 & NADES-1 & Colorless transparent oil \\
ChCl: Urea & $1: 2$ & 20 & NADES-1-W & Colorless transparent oil \\
ChCl: Glycerol & $1: 1$ & 0 & NADES-2 & Colorless transparent oil \\
ChCl: Glycerol & $1: 1$ & 20 & NADES-2-W & Colorless transparent oil \\
ChCl: Lactic acid & $1: 1$ & 0 & NADES-3 & Colorless transparent oil \\
ChCl: Lactic acid & $1: 1$ & 20 & NADES-3-W & Colorless transparent oil \\
ChCl: Ethylene glycol & $1: 1$ & 0 & NADES-4 & Colorless transparent oil \\
ChCl: Ethylene glycol & $1: 1$ & 20 & NADES-4-W & Colorless transparent oil \\
ChCl: Citric acid & $1: 1$ & 0 & NADES-5 & Pale yellow semisolid \\
ChCl: Citric acid & $1: 1$ & 20 & NADES-5-W & Pale yellow oil \\
\hline
\end{tabular}

The NADESs preparation was carried out by simply mixing the two components in a suitable manner and leaving under constant stirring at $80{ }^{\circ} \mathrm{C}$ for $2 \mathrm{~h}$ until a liquid was formed (Table 1 ). The prepared NADES were used without the need for purification as extraction solvents for phenolic compounds in olive leaves.

The viscosity of DESs is their main constraint, since it hinders the handling and efficiency as extraction solvents compared to conventional ones. Thus, the addition of water is often performed to adjust the properties of DES and decrease the viscosity and the surface tension. For this reason, a percentage of $20 \%$ of water $(w / w)$ was set for the screening extraction of the NADESs.

\subsection{Sample Preparation and Extraction Procedure}

Both fresh and properly dried leaves were extracted. The drying process is necessary to remove their water content, with the aim of protecting them from enzymatic degradation. This process was carried out keeping samples in a traditional oven at $80^{\circ} \mathrm{C}$ for $45 \mathrm{~min}$.

Then, the leaves were chopped to facilitate the entry of solvents into the cells and thus increase the efficiency of the extraction. The extraction was performed under microwave assistance to favor 
and accelerate the extraction of phenols present in the plant matrix. In fact, besides the use of green solvents, one of the criteria for the development of an eco-friendly extraction is to reduce energy consumption by using innovative technologies such as microwave-assisted extraction (MAE). MAE has been recognized as an exceptional energy resource for promoting extractions, increasing the yield, the quality of the product and greatly reducing the extraction time.

One of the main factors contributing to the efficiency of microwave extraction is the identification of the optimal extraction temperature [47]. In general, at high temperatures, the solvent power increases because there is a decrease in viscosity and diffusivity, which is very important for viscous solvents such as NADES. An increase in temperature can also cause a reduction in surface tension, as well as a decrease in the interaction between the target compound and the sample matrix, leading to an improvement in the desorption and dissolution of the target compound in the solvent. On the other hand, high temperatures can induce thermal degradation of phenolic compounds. Therefore, we decided to apply, in this study, moderate temperatures equal to $80^{\circ} \mathrm{C}$ and the microwave power did not exceed $800 \mathrm{~W}$. The maximum limit of the microwave power used was set in such a way as to avoid overheating of the extraction mixture and the consequent degradation of phenolic compounds.

An extraction time of $30 \mathrm{~min}$ is sufficient to obtain maximum yields of phenolic compounds, as well as to obtain a greater extraction of oleuropein in the classic aqueous extraction procedure [12]. Thus, for control, a conventional extraction in water was also performed.

In particular, MAE extraction was carried out by placing $2 \mathrm{~g}$ of fresh or dried leaves in a flask equipped with refrigerant in $8 \mathrm{~mL}$ of NADES at $80^{\circ} \mathrm{C}$ at a power of $800 \mathrm{~W}$. The comparison extraction was carried out by placing, in a flask equipped with coolant, $2 \mathrm{~g}$ of fresh or dried leaves in $8 \mathrm{~mL}$ of $\mathrm{H}_{2} \mathrm{O}$ at $80{ }^{\circ} \mathrm{C}$ at a power of $800 \mathrm{~W}$. The extracts were then centrifuged for $10 \mathrm{~min}$ and an aliquot of each extract was finally dissolved in ethanol and directly subjected to HPLC and LC-ESI-QTOF/MS analysis.

\subsection{HPLC-ESI-QTOF-MS Analysis and Quantitative HPLC}

The precise identification of the extracted phenolic compounds is not generally simple as different types of structures are contained. The HPLC-QTOF-MS is a very useful tool in order to characterize natural products. In particular, electrospray ionization (ESI) has been widely applied thanks to its mild ionization technique. An accurate measurement of the mass of small molecules is generally used to determine the elementary formulas, facilitating the identification of unknown substances. The QTOF-MS mass spectrometer combines high sensitivity and mass accuracy for both precursor and fragment ions, providing the elemental composition. This feature helped in the complete identification of the compounds and in the differentiation between the isobaric compounds. The ability of HPLC-ESI-QTOF-MS for qualitative identifications has been reported in several studies [48-50].

The UPLC profile of the phenolic compounds of the NADES leaf extracts using MAE are presented in Table 2 where the data obtained from the mass spectra of the identified compounds are summarized, including both the experimental $\mathrm{m} / \mathrm{z}$ values and those calculated for the molecular formulas, and the principal ions, as well as the proposed compound for each peak.

As shown in Table 2, the identified phenolic compounds are almost all derived from Oleuropein.

According to literature data, leaf extracts contain hydroxytyrosol, tyrosol, demethyloleuropein, oleuropein, and one of its isomers, oleacin, oleuropein aglycone (Table 2). It is worth noting that oleuropein is the main phenolic compound identified in all the extracts, and it was identified based on a comparison with the authentic standard. This compound has been described as the major component present in olive leaves. Oleuropein was found in olive leaves together with its isomer.

Another phenolic compound identified in olive tree leaf extracts is tyrosol (NADES-3, NADES-4, NADES-5), which, together with hydroxytyrosol, is in fact one of the best antioxidant phenolic compounds able to prevent natural oxidative processes. Additionally, the presence of the aglycone oleuropein was detected (NADES-2, NADES-3, NADES-4, NADES-5), while demethyloleuropein was detected in green leaves extracted with NADES-1, and in the case of dried leaves when the extraction was performed with NADES-4. 
Table 2. Compounds identified by UPLC- ESI-QTOF-MS analysis in olive leaf extracts extracted with NADESs.

\begin{tabular}{|c|c|c|c|c|c|c|c|}
\hline Compound & Htytrosol & Tyrosol & Demethyloleuropein & Oleuropein & 3,4-DHPEA-EDA & Isomer Oleuropein & 3,4-DHPEA-EA \\
\hline r.t. $\min$ & 2.47 & 2.95 & 3.36 & 3.89 & 3.97 & 4.03 & 5.10 \\
\hline Formula & $\mathrm{C}_{8} \mathrm{H}_{10} \mathrm{O}_{3}$ & $\mathrm{C}_{8} \mathrm{H}_{10} \mathrm{O}_{2}$ & $\mathrm{C}_{24} \mathrm{H}_{30} \mathrm{O}_{13}$ & $\mathrm{C}_{25} \mathrm{H}_{32} \mathrm{O}_{13}$ & $\mathrm{C}_{17} \mathrm{H}_{20} \mathrm{O}_{6}$ & $\mathrm{C}_{25} \mathrm{H}_{32} \mathrm{O}_{13}$ & $\mathrm{C}_{19} \mathrm{H}_{22} \mathrm{O}_{8}$ \\
\hline $\begin{array}{c}\text { Theorical } \\
m / z\end{array}$ & 154.0630 & 138.0681 & 526.1686 & 540.1843 & 320.1260 & 540.1843 & 378.1315 \\
\hline $\begin{array}{c}\text { Measured } \\
m / z\end{array}$ & 154.0634 & 138.0673 & 526.1670 & 540.1828 & 320.1253 & 540.1833 & 378.1309 \\
\hline $\begin{array}{l}\text { MS spectrum } \\
\text { peak list }\end{array}$ & $177.0511(\mathrm{M}+\mathrm{Na})^{+}$ & $\begin{array}{c}139.0744(\mathrm{M}+\mathrm{H})^{+} \\
161.0576(\mathrm{M}+\mathrm{Na})^{+}\end{array}$ & $\begin{array}{c}527.1760(\mathrm{M}+\mathrm{H})^{+} \\
549.1557(\mathrm{M}+\mathrm{Na})^{+}\end{array}$ & $563.1726(\mathrm{M}+\mathrm{Na})^{+}$ & $343.1138(\mathrm{M}+\mathrm{H})^{+}$ & $563.1727(\mathrm{M}+\mathrm{Na})^{+}$ & $401.1200(\mathrm{M}+\mathrm{Na})^{+}$ \\
\hline Fresh Leaves & $\begin{array}{l}\text { NADES-4, } \\
\text { NADES-5 }\end{array}$ & $\begin{array}{l}\text { NADES-3, } \\
\text { NADES-4, } \\
\text { NADES-5 }\end{array}$ & NADES-1 & $\begin{array}{l}\text { NADES-1, } \\
\text { NADES-2, } \\
\text { NADES-3, } \\
\text { NADES-4 }\end{array}$ & $\begin{array}{l}\text { NADES-3, } \\
\text { NADES-4, } \\
\text { NADES-5 }\end{array}$ & $\begin{array}{l}\text { NADES-1, } \\
\text { NADES-2, } \\
\text { NADES-3, } \\
\text { NADES-4 }\end{array}$ & $\begin{array}{l}\text { NADES-2, } \\
\text { NADES-3, } \\
\text { NADES-4, } \\
\text { NADES-5 }\end{array}$ \\
\hline Dried Leaves & $\begin{array}{l}\text { NADES-2, } \\
\text { NADES-4, } \\
\text { NADES-5 }\end{array}$ & $\begin{array}{l}\text { NADES-2, } \\
\text { NADES-3 }\end{array}$ & NADES-4 & $\begin{array}{l}\text { NADES-2, } \\
\text { NADES-3, } \\
\text { NADES-4 }\end{array}$ & & $\begin{array}{l}\text { NADES-2, } \\
\text { NADES-3, } \\
\text { NADES-4 }\end{array}$ & NADES-2 \\
\hline
\end{tabular}


The same extracts were then analyzed by HPLC and the polyphenol content was quantified by comparison with the standards. The results obtained, reported in Table 3, once again show that, as expected, oleuropein is the most abundant component present in olive leaves and that the other components identified with UPLC-MS are instead present in traces.

Table 3. Content of phenolic compounds in the examined olive leaf extracts (in NADESs or water) from HPLC analysis ${ }^{a}$.

\begin{tabular}{clc}
\hline Solvent & Fresh Leaves & $\begin{array}{c}\text { Oleuropein } \\
\text { [ppm] }\end{array}$ \\
\hline \multirow{2}{*}{ NADES-1 } & Dried Leaves & ND \\
& Fresh Leaves & $333.73 \pm 0.17$ \\
NADES-2 & Dried Leaves & $416.08 \pm 0.15$ \\
& Fresh Leaves & $86.30 \pm 1.12$ \\
NADES-3 & Dried Leaves & $126.51 \pm 0.10$ \\
& Fresh Leaves & ND \\
NADES-4 & Dried Leaves & $72.97 \pm 0.05$ \\
& Fresh Leaves & ND \\
NADES-5 & Dried Leaves & ND \\
& Fresh Leaves & ND \\
Water & Dried Leaves & $174.47 \pm 0.42$ \\
\hline
\end{tabular}

${ }^{a}$ Htyrosol, tyrosol, demethyloleuropein, 3,4-DHPEA-EA, 3,4-DHPEA-EDA are in an amount not detectable by HPLC. ${ }^{b}$ Data are expressed as the means \pm SD of three independent observations. $(p<0.05)$. ND $=$ not detected.

Generally, the extraction process is greatly affected by the affinity of the DES with the target compounds [51]. In fact, glycerol-based NADES-2 evidenced to be the best solvent for the extraction of oleuropein, followed by NADES-3 and NADES-4. The urea-based NADES and the citric acid-based NADES were discarded due to their low extraction efficiency. The results of the extraction yields are also in agreement with what was predictable based on the viscosity: in fact, the NADES with glycerol, lactic acid are the most efficient and have the lowest viscosity (29.5 and $47.5 \mathrm{cP}$, respectively), while the other NADES values have higher viscosity values, e.g., NADES with citric acid has viscosity $=448.1 \mathrm{cP}$ ) [52]. In the case, instead, of NADES based on urea, the low extraction efficiency is probably due to the lower polarity of NADES compared to those containing polyols or organic acids.

To compare the extraction efficiency of the methodology based on NADES under microwave assistance with the conventional extraction method, a MAE extraction with water was carried out according to protocols already reported in the literature.

The obtained water extract was centrifuged and dried under reduced pressure conditions using a rotary evaporator. The sample was then diluted with the appropriate solvent for the subsequent chromatographic analysis. The phenolic compounds identified and quantified by HPLC in the aqueous extract are oleuropein, as the main component, and traces of hydroxytyrosol and oleacein (Table 3). The construction of a histogram for oleuropein makes it possible to compare the extraction efficiency of the NADESs with that of water (Figure 2).

It is evident from the histogram that NADES-2 consisting of choline chloride and glycerol as a hydrogen bond donor was not only the best among the tested NADESs, but also the green solvent with superior extraction capacity with respect to water $(8.31 \pm 0.11 \mathrm{ppm}$ versus $5.20 \pm 0.10 \mathrm{ppm})$.

Thus, we decided to apply the same procedure for the extraction of phenolic compounds from ripe olives. The MAE process was performed for 10 and for $30 \mathrm{~min}$ in order to validate the procedure, while at the same time verifying the ability of the NADES to convert oleuropein into demethyloleuropein (already present in ripe olives due to the enzymatic activity during ripening), its aglyconic form (3,4-DHPEA-EDA), and hydroxytyrosol. Table 4 reports the phenolic compounds present in the NADES extracts, as well as the phenolics present in water extract. 


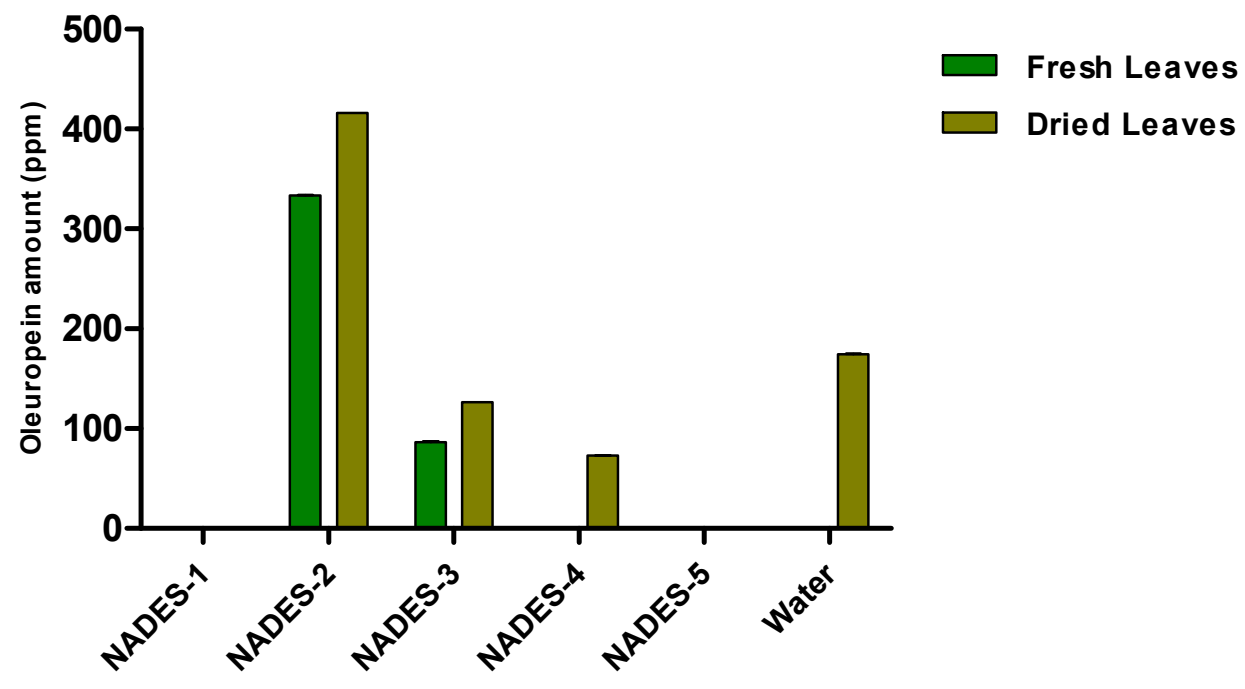

Figure 2. Quantitative analysis of oleuropein in olive leaf extracts carried out by HPLC. Data are expressed as mean \pm SD of three independent observations $(p<0.05)$.

Table 4. Content of phenolic compounds in the examined ripe olive extracts (in NADESs or water) from HPLC analysis ${ }^{\text {a }}$.

\begin{tabular}{ccccc}
\hline Solvent & Time (min) & $\begin{array}{c}\text { Demethyloleuropein } \\
{[\mathbf{p p m}]^{\mathbf{b}}}\end{array}$ & $\begin{array}{c}\text { 3,4-DHPEA-EDA } \\
{[\mathbf{p p m}]^{\mathbf{b}}}\end{array}$ & $\begin{array}{c}\text { Oleuropein } \\
\text { [ppm] }^{\mathbf{b}}\end{array}$ \\
\hline NADES-1 & 10 & $\mathrm{ND}$ & $\mathrm{ND}$ & $\mathrm{ND}$ \\
& 30 & $\mathrm{ND}$ & $\mathrm{ND}$ & $\mathrm{ND}$ \\
NADES-2 & 10 & $284.67 \pm 1.13$ & $156.64 \pm 1.81$ & $88320.90 \pm 38.03$ \\
& 30 & $1019.84 \pm 0.53$ & $480.60 \pm 0.55$ & $37602.42 \pm 1.21$ \\
NADES-3 & 10 & $1918.761 \pm 1.88$ & $\mathrm{ND}$ & $52643.39 \pm 51.7$ \\
& 30 & $4527.07 \pm 18.19$ & $193.15 \pm 0.55$ & $23338.72 \pm 10.50$ \\
NADES-4 & 10 & $1655.97 \pm 2.03$ & $420.92 \pm 1.92$ & $\mathrm{ND}$ \\
NADES-5 & 30 & $903.79 \pm 2.15$ & $\mathrm{ND}$ & $\mathrm{ND}$ \\
& 10 & $\mathrm{ND}$ & $637.06 \pm 1.74$ & $\mathrm{ND}$ \\
Water & 30 & $174.95 \pm 0.93$ & $25.69 \pm 1.73$ & $42534.86 \pm 12.12$ \\
& 10 & $4000.52 \pm 2.15$ & $503.05 \pm 1.31$ & $\mathrm{ND}$ \\
& 30 & $5602.64 \pm 8.72$ & $783.25 \pm 3.23$ & $\mathrm{ND}$ \\
\hline
\end{tabular}

${ }^{a}$ Htyrosol, tyrosol, 3,4-DHPEA-EDA are in an amount not detectable by HPLC. ${ }^{b}$ Data are expressed as mean \pm SD based on triplicate values $(p<0.05)$. ND = Not Detected.

Even in this case, the construction of histograms for oleuropein, demethyloleuropein and 3,4-DHPEA-EDA amounts allowed us to make a visual comparison between the extraction efficiency of NADESs with that of water (Figure 3).

For all the extracts, the amount of detected hydroxytyrosol is below the limit of detection. Prolonged extraction times resulted in an increase in the amount of phenolic compounds, other than some small discrepancies. When NADES-2 and NADES-3 were used as solvents, an oleuropein-rich extract was obtained after only $10 \mathrm{~min}$. The maximum increase in the yield of oleuropein was surprising in the case of NADES-2 (88287.57 \pm 0.24 ppm of oleuropein) as it was a yield that practically doubled that obtained with a conventional water extraction carried out for $30 \mathrm{~min}$.

In addition, a decrease of oleuropein content over time (after $30 \mathrm{~min}$ ) was observed with a concomitant increase of the amount of demethyloleuropein. Excellent results were obtained in the extraction process of demethyloleuropein using NADES-3 after 30 min of extraction.

In the final part of our study, we performed some experiments to assess the extraction efficiency of NADES with the addition of a certain quantity of water. The high viscosity of NADES would limit the transfer of the target compound from the natural matrix. 


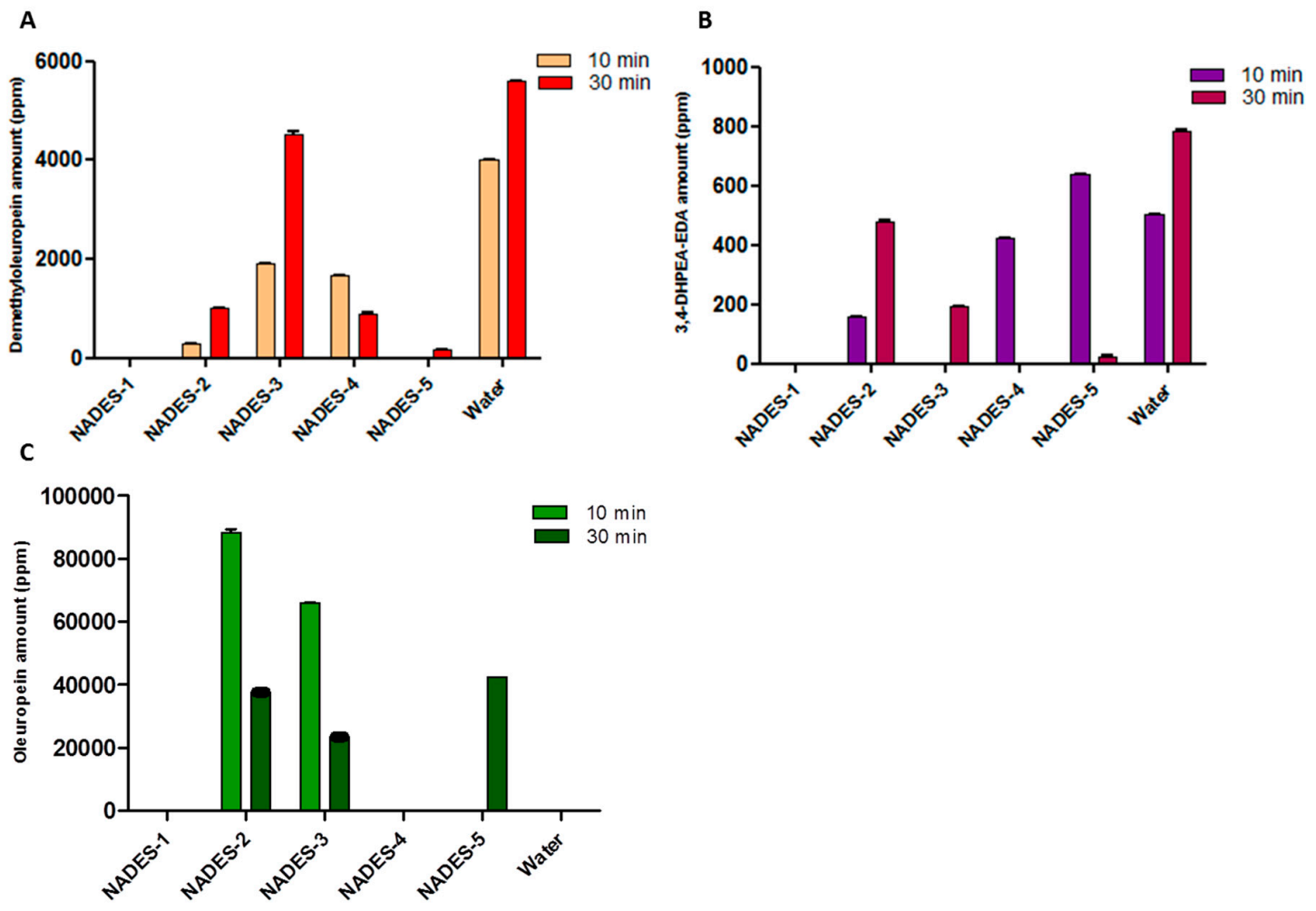

Figure 3. Quantitative analysis, carried out by HPLC, of demethyloleuropein (A), 3,4-DHPEA-EDA (B) and oleuropein $(C)$ in ripe olives extracted with NADESs and water (for control). The extractions were carried out for a duration of 10 and of $30 \mathrm{~min}$.

The water addition is a pivotal factor to reduce the DES's viscosity since the interactions (e.g., hydrogen bonding) between the components of the DES are weaken in presence of water. To tackle this issue, we attempted the extraction using aqueous solutions of NADES. According to the literature, a percentage of $20 \%$ water content [53] can sufficiently reduce the viscosity of the NADES and meanwhile maintain the hydrogen-bonding network. Higher amount of water could gradually weaken the interactions between the components of the DESs.

This test was carried out by extracting the ripe olive samples with the NADES added with $20 \%(w / w)$ of water. All of the tested NADES, except for NADES-1, were only able to extract demethyloleuropein and 3,4-DHPEA-EDA (Table 5).

Table 5. Content of phenolic compounds extracted in the ripe olives (in NADES added with 20\% water) from HPLC analysis ${ }^{\text {a }}$.

\begin{tabular}{|c|c|c|c|}
\hline Solvent & $\begin{array}{l}\text { Time } \\
\text { min. }\end{array}$ & $\begin{array}{l}\text { Demethyloleuropein } \\
\text { [ppm] }^{\mathbf{b}}\end{array}$ & $\begin{array}{l}\text { 3,4-DHPEA-EDA } \\
\text { [ppm] }^{b}\end{array}$ \\
\hline \multirow{2}{*}{ NADES-1-W } & 10 & ND & ND \\
\hline & 30 & ND & ND \\
\hline \multirow{2}{*}{ NADES-2-W } & 10 & $1309.518 \pm 2.124$ & $198.3333 \pm 0.994$ \\
\hline & 30 & $869.8605 \pm 3.384$ & $254.4684 \pm 1.384$ \\
\hline \multirow{2}{*}{ NADES-3-W } & 10 & $567.562 \pm 1.158$ & $469.912 \pm 2.143$ \\
\hline & 30 & $338.0844 \pm 2.964$ & $438.5344 \pm 1.951$ \\
\hline \multirow{2}{*}{ NADES-4-W } & 10 & $303.2958 \pm 3.121$ & $154.5307 \pm 1.005$ \\
\hline & 30 & $241.5935 \pm 4.001$ & $105.9052 \pm 1.805$ \\
\hline \multirow{2}{*}{ NADES-5-W } & 10 & $1303.292 \pm 2.021$ & ND \\
\hline & 30 & $2125.9 \pm 1.976$ & ND \\
\hline
\end{tabular}

${ }^{a}$ Htyrosol, Oleuropein, 3,4-DHPEA-EA are in an amount not detectable by HPLC. ${ }^{b}$ Data are expressed as mean \pm $\mathrm{SD}$ of three independent observations $(p<0.05)$. ND = Not Detected. 
Actually, quantitative analyses on all the extracts obtained from the new NADESs do not reveal the presence of oleuropein and, anyhow, the quantity of demethyloleuropein determined is lower than that determined in the classic aqueous extract. However, the extracts obtained using NADES-2-W and NADES-3-W show the presence of 3,4-DHPEA-EDA, which is not identified in the classic aqueous extraction (water). Furthermore, once again NADES-2-W, which has glycerol as its component, shows that after $30 \mathrm{~min}$ of extraction, the quantity of demethyloleuropein decreases, while the quantity of 3,4-DHPEA-EDA increases (compared to the quantities detected after $10 \mathrm{~min}$ extraction).

This result leads us to believe that NADES-2-W, in addition to the function of extraction solvent, favors the formation of oleacein (3,4-DHPEA-EDA) from demethyloleuropein through the synthetic transformation already reported [18].

\section{Conclusions}

The results presented in this work proved that the combination of sustainable green solvents (NADESs) and microwave assisted extraction techniques (MAE) is an efficient approach for recovering phenolic compounds from olive oil processing wastes.

Phenolic compounds are polar molecules and the differences observed in the extraction efficiency of various NADESs could be explained because their chemical and physical properties are determined by their chemical form. In general, the functional groups involved in the hydrogen bonds are the hydroxyl, amidic and carboxylic groups which are abundant in NADES and are obviously present in phenolic compounds. H-bonding interactions between molecules of NADES and phenolic compounds are responsible for their extractability.

The urea-based NADES-1 was discarded due to its poor power of extraction. The polyols-based NADESs, NADES-2 and NADES-4, as well as organic acid-based NADES-3, proved to be effective in the extraction of phenolic compounds from olive leaves and drupes. Glycerol-based NADES-2 was confirmed to be an excellent solvent, and was even more effective than conventional solvent.

The proposed NADES-based microwave-assisted extraction process is highly efficient and really eco-friendly. The natural origin of the components of the NADES makes them attractive for further applications in cosmetics, pharmaceuticals and food industries. The NADES extract could be used in their wholeness, even in products to be used or consumed by people.

Supplementary Materials: The following are available online at http://www.mdpi.com/2076-3921/9/6/513/s1.

Author Contributions: Conceptualization, M.L.D.G. and P.C.; Data curation, L.M.; Formal analysis, S.T.; Investigation, S.B.; Project administration, M.L.D.G. and P.C.; Supervision, M.N.; Writing-review \& editing, M.L.D.G and M.N. All authors have read and agreed to the published version of the manuscript.

Funding: This research received no external funding.

Conflicts of Interest: The authors declare no conflict of interest.

\section{References}

1. Silva, S.; Gomes, L.; Leitao, F.; Leitao, F.; Coelho, A.V.; Vilas Boas, L.; Phenolic compounds and antioxidant activity of Olea europaea L. fruits and leaves. Food Sci. Technol. Int. 2006, 12, 385-395. [CrossRef]

2. Kountouri, A.M.; Mylona, A.; Kaliora, A.C.; Andrikopoulos, N.K. Bioavailability of the phenolic compounds of the fruits (drupes) of Olea europaea (olives): impact on plasma antioxidant status in humans. Phytomedicine 2007, 14, 659-667. [CrossRef] [PubMed]

3. Owen, R.W.; Mier, W.; Giacosa, A.; Hull, W.E.; Spiegelhalder, B.; Bartsch, H. Identification of lignans as a major component in the phenolic fraction of olive oil. Clin. Chem. 2000, 46, 976-988. [CrossRef] [PubMed]

4. Hassen, I.; Casabianca, H.; Hosni, K. Biological activities of the natural antioxidant oleuropein: Exceeding the expectation-A mini-review. J. Funct. Foods 2015, 18, 926-940. [CrossRef]

5. Angeloni, C.; Malaguti, M.; Barbalace, M.C.; Hrelia, S. Bioactivity of Olive Oil Phenols in Neuroprotection. Int. J. Mol. Sci. 2017, 18, 2230. [CrossRef] [PubMed] 
6. Bouaziz, M.; Fki, I.; Jemai, H.; Ayadi, M.; Sayadi, S. Effect of storage on refined and husk olive oils composition: Stabilization by addition of natural antioxidants from Chemlali olive leaves. Food Chem. 2008, 108, $253-262$. [CrossRef]

7. Pereira, A.P.; Ferreira, I.C.; Marcelino, F.; Valentão, P.; Andrade, P.B.; Seabra, R.; Estevinho, L.; Bento, A.; Pereira, J.A. Phenolic compounds and antimicrobial activity of olive (Olea europaea L. Cv. Cobrançosa) leaves. Molecules 2007, 12, 1153-1162. [CrossRef] [PubMed]

8. Lídia, A.; Cavaca, S.; Afonso, C.A.M. Oleuropein: A Valuable Bio-Renewable Synthetic Building Block. Eur. J. Org. Chem. 2018, 581-589.

9. Procopio, A.; Sindona, G.; Gaspari, M.; Costa, N.; Nardi, M. Chemical-Catalytic Method for the Peracylation of Oleuropein and Its Products of Hydrolysis. Patent WO2008136037 A2, 24 December 2008.

10. El Riachy, M.; Priego-Capote, F.; Leon, L.; Rallo, L.; Dolores, M.; de Castro, L. Hydrophilic antioxidants of virgin olive oil. Part 2: Biosynthesis and biotransformation of phenolic compounds in virgin olive oil as affected by agronomic and processing factors. Eur. J. Lipid Sci. Tech. 2011, 113, 692-707. [CrossRef]

11. Procopio, A.; Alcaro, S.; Nardi, M.; Oliverio, M.; Paolini, D.; Sindona, G. Lipophilic hydroxytyrosol esters: fatty acid conjugates for potential topical administration. J. Nat. Prod. 2011, 74, 2377-2381. [CrossRef] [PubMed]

12. Procopio, A.; Alcaro, S.; Nardi, M.; Oliverio, M.; Paolini, D.; Sindona, G. Synthesis, biological evaluation, and molecular modeling of oleuropein and its semisynthetic derivatives as cyclooxygenase inhibitors. J. Agric. Food Chem. 2009, 57, 11161-11167. [CrossRef] [PubMed]

13. Beauchamp, G.K.; Keast, R.S.J.; Morel, D.; Lin, J.; Pika, J.; Han, Q.; Lee, C.-H.; Smith, A.B.; Breslin, P.A.S. Phytochemistry: ibuprofen-like activity in extra-virgin olive oil. Nature 2005, 437, 45-46. [CrossRef] [PubMed]

14. Breslin, P.A.S.; Gingerich, T.N.; Green, B.G. Ibuprofen as a chemesthetic stimulus: evidence of a novel mechanism of throat irritation. Chem. Sens. 2001, 26, 55-66. [CrossRef] [PubMed]

15. Sindona, G.; Caruso, A.; Cozza, A.; Fiorentini, S.; Lorusoo, B.; Marini, E.; Nardi, M.; Procopio, A.; Zicari, S. Antiinflammatory effect of 3,4-DHPEA-EDA [2-(3,4 -hydroxyphenyl) ethyl (3S, 4E)-4-formyl3-(2-oxoethyl)hex-4-enoate] on primary human vascular endothelial cells. Curr. Med. Chem. 2012, 19, 4006-4013.

16. Parzonko, A.; Czerwińska, M.E.; Kiss, A.K.; Naruszewicz, M. Oleuropein and oleacein may restore biological functions of endothelial progenitor cells impaired by angiotensin II via activation of Nrf2/heme oxygenase pathway. Phytomedicine 2013, 20, 1088-1094. [CrossRef] [PubMed]

17. Czerwin'ska, M.E.; Kiss, K.A.; Naruszewicz, M. Inhibition of human neutrophils NEP activity, CD11b/CD18 expression and elastase release by 3, 4-dihydroxyphenylethanol-elenolic acid dialdehyde, oleacein. Food Chem. 2014, 153, 1-8. [CrossRef] [PubMed]

18. Nardi, M.; Bonacci, S.; De Luca, G.; Maiuolo, J.; Oliverio, M.; Sindona, G.; Procopio, A. Biomimetic synthesis and antioxidant evaluation of 3,4-DHPEA-EDA [2-(3,4-hydroxyphenyl) ethyl (3S,4E)-4-formyl3-(2-oxoethyl)hex-4-enoate]. Food Chem. 2014, 124, 89-93. [CrossRef] [PubMed]

19. Costanzo, P.; Bonacci, S.; Cariati, L.; Nardi, M.; Oliverio, M.; Procopio, A. Simple and efficient sustainable semi-synthesis of oleacein [2-(3,4-hydroxyphenyl) ethyl (3S,4E)-4-formyl-3-(2-oxoethyl)hex-4-enoate] as potential additive for edible oils. Food Chem. 2018, 245, 410-414. [CrossRef] [PubMed]

20. Lozano-Castellón, J.; López-Yerena, A.; Rinaldi de Alvarenga, J.F.; Romero del Castillo-Alba, J.; VallverdúQueralta, A.; Escribano-Ferrer, E.; Lamuela-Raventósa, R.M. Health-promoting properties of oleocanthal and oleacein: Two secoiridoids from extra-virgin olive oil. Crit. Rev. Food Sci. Nutr. 2019, 1-17. [CrossRef]

21. Pang, K.-L.; Chin, K.-Y. The Biological Activities of Oleocanthal from a Molecular Perspective. Nutrients 2018, 10, 570. [CrossRef] [PubMed]

22. Ryan, D.; Robards, K.; Lavee, S. Changes in phenolic content of olive during maturation. Int. J. Food Sci. Tech. 1999, 34, 265-274. [CrossRef]

23. Smith, A.B.; Han, Q.; Breslin, P.A.S.; Beauchamp, G.K. Synthesis and assignment of absolute configuration of (-)-oleocanthal: A potent, naturally occurring non-steroidal anti-inflammatory and anti-oxidant agent derived from extra virgin olive oils. Org. Lett. 2005, 7, 5075-5078. [CrossRef] [PubMed]

24. Smith, A.B.; Sperry, J.B.; Han, Q. Syntheses of (-)-oleocanthal, a natural NSAID found in extra virgin olive oil, the (-)-deacetoxy-oleuropein aglycone, and related analogues. J. Org. Chem. 2007, 72, 6891-6900. [CrossRef] [PubMed] 
25. English, B.J.; Williams, R.M. Synthesis of (+/-)-oleocanthal via a tandem intramolecular Michael cyclization-HWE olefination. Tetrahedron Lett. 2009, 50, 2703-2840. [CrossRef] [PubMed]

26. Takahashi, K.; Morita, H.; Honda, T. Formal synthesis of (-)-oleocanthal by means of a SmI2-promoted intramolecular coupling of bromoalkyne with $\alpha, \beta$-unsaturated ester. Tetrahedron Lett. 2012, 53, 3342-3345. [CrossRef]

27. Ross, K.; Beta, T.; Arntfield, S. A comparative study on the phenolic acids identified and quantified in dry beans using HPLC as affected by different extraction and hydrolysis methods. Food Chem. 2009, 113, 336-344. [CrossRef]

28. Cravotto, G.; Abert Vian, M.; Chemat, F. Green Extraction of Natural Products: Concept and Principles. Int. J. Mol. Sci. 2012, 13, 8615-8627.

29. Chemat, F.; Vian, M.A.; Fabiano-Tixier, A.S.; Nutrizio, M.; Jambrak, A.R.; Munekata, P.E.S.; Lorenzo, J.M.; Barba, F.J.; Binelloe, A.; Cravotto, G. A review of sustainable and intensified techniques for extraction of food and natural products. Green Chem. 2020, 22, 2325. [CrossRef]

30. Cvjetko Bubalo, M.; Vidović, S.; Radojčić Redovniković, I.; Jokić, S. Green solvents for green technologies. J. Chem. Technol. Biotechnol. 2015, 90, 1631-1639. [CrossRef]

31. Paiva, P.; Craveiro, R.; Aroso, I.; Martins, M.; Reis, R.L.; Duarte, A.R.C. Natural deep eutectic solvents-solvents for the 21st century. Acs Sustain. Chem. Eng. 2014, 2, 1063-1071. [CrossRef]

32. Di Gioia, M.L.; Cassano, R.; Costanzo, P.; Herrera Cano, N.; Maiuolo, L.; Nardi, M.; Nicoletta, F.P.; Oliverio, M.; Procopio, A. Green Synthesis of Privileged Benzimidazole Scaffolds Using Active Deep Eutectic Solvent. Molecules 2019, 24, 2885. [CrossRef] [PubMed]

33. Di Gioia, M.L.; Nardi, M.; Costanzo, P.; De Nino, A.; Maiuolo, L.; Oliverio, M.; Procopio, A. Biorenewable deep eutectic solvent for selective and scalable conversion of furfural into cyclopentenone derivatives. Molecules 2018, 23, 1891. [CrossRef] [PubMed]

34. Di Gioia, M.L.; Costanzo, P.; De Nino, A.; Maiuolo, L.; Nardi, M.; Olivito, F.; Procopio, A. Simple and efficient Fmoc removal in ionic liquid. RSC Adv. 2017, 7, 36482-36491. [CrossRef]

35. Perna, F.M.; Vitale, P.; Capriati, V. Deep eutectic solvents and their applications as green solvents. Curr. Opin. Green Sustain. Chem. 2020, 21, 27-33. [CrossRef]

36. Gertrudes, A.; Craveiro, R.; Eltayari, Z.; Reis, R.L.; Paiva, A.; Duarte, A.R.C. How Do Animals Survive Extreme Temperature Amplitudes? The Role of Natural Deep Eutectic Solvents. ACS Sustain. Chem. Eng. 2017, 5, 9542-9553. [CrossRef]

37. Radošević, K.; Ćurko, N.; Srček, V.G.; Bubalo, M.C.; Tomašević, M.; Ganić, K.K.; Redovniković, I.R. Natural deep eutectic solvents as beneficial extractants for enhancement of plant extracts bioactivity. LWT Food Sci. Technol. 2016, 73, 45. [CrossRef]

38. Young, H.C.; van Spronsen, J.; Dai, Y.; Verberne, M.; Hollmann, F.; Arends Isabel, W.C.E.; Verpoorte, R. Are natural deep eutectic solvents the missing link in understanding cellular metabolism and physiology? Plant Physiol. 2011, 156, 1701-1705.

39. Francisco, M.; Van den Bruinhorst, A.; Kroon, M. Low-Transition-Temperature Mixtures (LTTMs): A New Generation of Designer Solvents. Angew. Chem. Int. Edit. 2013, 52, 3074-3085. [CrossRef] [PubMed]

40. Henni, V.; Dai, Y.; Wilson, E.G.; Verpoorte, R.; Choi, Y.H.; Verpoorte, R. Green solvents from ionic liquids and deep eutectic solvents to natural deep eutectic solvents. C. R. Chim. 2018, 21, 628-638.

41. Chemat, F.; Abert Vian, M.; Ravi, H.K.; Khadhraoui, B.; Hilali, S.; Perino, S.; Fabiano, A.S.T. Review of Alternative Solvents for Green Extraction of Food and Natural Products: Panorama, Principles, Applications and Prospects. Molecules 2019, 24, 3007. [CrossRef] [PubMed]

42. Daia, Y.; van Spronsenb, J.; Witkamp, G.J.; Verpoorte, R.; Choia, Y.H. Natural deep eutectic solvents as new potential media for green Technology. Anal. Chim. Acta 2013, 766, 61-68. [CrossRef] [PubMed]

43. Lakka, A.; Grigorakis, S.; Karageorgou, I.; Batra, G.; Kaltsa, O.; Bozinou, E.; Lalas, S.; Makris, D.P. Saffron Processing Wastes as a Bioresource of High-Value Added Compounds: Development of a Green Extraction Process for Polyphenol Recovery Using a Natural Deep Eutectic Solvent. Antioxidants 2019, 8, 586. [CrossRef] [PubMed]

44. García, A.; Rodríguez-Juan, E.; Rodríguez-Gutiérrez, G.; Rios, J.J.; Fernández-Bolaños, J. Extraction of phenolic compounds from virgin olive oil by deep eutectic solvents (DESs). Food Chem. 2016, 197, 554-561. 
45. Ruesgas-Ramón, M.; Figueroa-Espinoza, M.C.; Durand, E. Application of Deep Eutectic Solvents (DES) for Phenolic Compounds Extraction: Overview, Challenges, and Opportunities. J. Agric. Food Chem. 2017, 65, 3591-3601. [CrossRef] [PubMed]

46. Gu, T.; Zhang, M.; Tan, T.; Chen, J.; Li, Z.; Zhang, Q.; Qiu, H. Deep eutectic solvents as novel extraction media for phenolic compounds from model oil. Chem. Commun. 2014, 50, 11749-11752. [CrossRef] [PubMed]

47. Rafiee, Z.; Jafari, S.M.; Alami, M.; Khomeiri, M. Microwave-assisted extraction of phenolic compounds from olive leaves; a comparison with maceration. J. Anim. Plant Sci. 2011, 21, 738-745.

48. Li, H.; Yao, W.; Liu, Q.; Xu, J.; Bao, B.; Shan, M.; Cao, Y.; Cheng, F.; Ding, A.; Zhang, L. Application of UHPLCESI-Q-TOF-MS to identify multiple constituents in processed products of the herbal medicine Ligustri Lucidi Fructus. Molecules 2017, 22, 689. [CrossRef] [PubMed]

49. Tzima, K.; Brunton, N.P.; Rai, D.K. Qualitative and quantitative analysis of polyphenols in Lamiaceae plants-A Review. Plants 2018, 7, 25. [CrossRef] [PubMed]

50. Abu-Reidah, I.M.; Arráez-Román, D.; Quirantes-Piné, R.; Fernández-Arroyo, S.; Segura-Carretero, A.; Fernández-Gutiérrez, A. HPLC-ESI-Q-TOF-MS for a comprehensive characterization of bioactive phenolic compounds in cucumber whole fruit extract. Food Res. Int. 2012, 46, 108-117. [CrossRef]

51. Yao, X.-H.; Zhang, D.-Y.; Duan, M.-H.; Cui, Q.; Xu, W.-J.; Luo, M.; Li, C.-Y.; Zu, Y.-G.; Fu, Y.-J. Preparation and determination of phenolic coumpounds from Pyrola Incarnata Fisch. with a green polyols based-deep eutectic solvent. Sep. Purif. Technol. 2015, 149, 116-123. [CrossRef]

52. Chanioti, S.; Tzia, C. Extraction of phenolic compounds from olive pomace by using natural deep eutectic solvents and innovative extraction techniques. Innov. Food Sci. Emerg. 2018, 48, 228-239. [CrossRef]

53. Dai, Y.; Witkamp, G.J.; Verpoorte, R.; Choi, Y.T. Tailoring properties of natural deep eutectic solvents with water to facilitate their applications. Food Chem. 2015, 187, 14-19. [CrossRef] [PubMed]

(C) 2020 by the authors. Licensee MDPI, Basel, Switzerland. This article is an open access article distributed under the terms and conditions of the Creative Commons Attribution (CC BY) license (http://creativecommons.org/licenses/by/4.0/). 